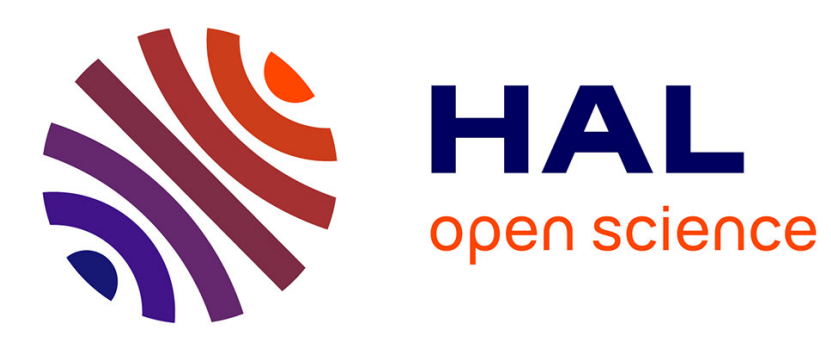

\title{
Terahertz beats of vibrational modes studied by femtosecond coherent Raman spectroscopy
}

\author{
R. Leonhardt, W. Holzapfel, W. Zinth, W. Kaiser
}

\section{To cite this version:}

R. Leonhardt, W. Holzapfel, W. Zinth, W. Kaiser. Terahertz beats of vibrational modes studied by femtosecond coherent Raman spectroscopy. Revue de Physique Appliquée, 1987, 22 (12), pp.17351741. 10.1051/rphysap:0198700220120173500 . jpa-00245733

\section{HAL Id: jpa-00245733 https://hal.science/jpa-00245733}

Submitted on 1 Jan 1987

HAL is a multi-disciplinary open access archive for the deposit and dissemination of scientific research documents, whether they are published or not. The documents may come from teaching and research institutions in France or abroad, or from public or private research centers.
L'archive ouverte pluridisciplinaire HAL, est destinée au dépôt et à la diffusion de documents scientifiques de niveau recherche, publiés ou non, émanant des établissements d'enseignement et de recherche français ou étrangers, des laboratoires publics ou privés. 


\title{
Terahertz beats of vibrational modes studied by femtosecond coherent Raman spectroscopy
}

\author{
R. Leonhardt, W. Holzapfel, W. Zinth and W. Kaiser \\ Physik Department Ell der Technischen Universität München, München, F.R.G.
}

(Reçu le 9 juin 1987, accepté le 18 septembre 1987)

\begin{abstract}
Résumé. - Une technique par Raman cohérent femtoseconde récemment développée permet de mesurer des spectres Raman cohérent à transformée de Fourier avec des différences haute-fréquence. L'excitation simultanée de différents modes de vibration avec une force motrice accordable à large bande conduit à un fort battement de la diffusion Raman cohérent de la sonde. La haute résolution temporelle du montage expérimental permet de mesurer des battements de fréquence de plus de $10 \mathrm{THz}$ avec une précision élevée.

Abstract. - A recently developed femtosecond coherent Raman technique allows the measurement of Fourier transform coherent Raman spectra with high-frequency differences. The simultaneous excitation of different vibrational modes with a broad-band tunable driving force leads to a strong beating of the coherent Raman probe scattering. The high time resolution of the experimental set-up allows one to measure beat frequencies of more than $10 \mathrm{THz}$ with high precision.
\end{abstract}

\section{Introduction.}

During the past decade, time-resolved coherent Raman methods have attracted considerable interest for the study of fast dynamic processes. In particular, time-resolved coherent Raman scattering made it possible to measure - in the time domain - rapid dephasing processes of molecular vibrations in liquids and elucidated various line broadening mechanisms [1-5]. It is the aim of this paper to focus attention on a more recent application. Although time-resolved Raman scattering collects data in the time domain, it allows valuable information to be obtained in the frequency domain. There are various approaches to relate the time and the frequency domain [6-11] : (i) Time resolved coherent Raman scattering, where the spectrum of the coherently scattered light is recorded, has been extensively studied using picosecond light pulses. It is possible to remove the homogeneous contribution to the transition line-width. One finds spectra with narrower lines and one obtains transition frequencies with high accuracy. This technique has been applied to the study of congested spectral regions, where the spontaneous Raman spectrum is smooth, but where the line-narrowing technique revealed structure due to distinct transitions [9-11]. (ii) In the second method the time evolution of the coherent Raman probe signal is recorded with high time resolution.
The Fourier transformation of the experimental data gives a difference frequency spectrum. The frequency resolution of this technique can be improved by using numerical filtering procedures prior to the Fourier transformation. (iii) A third way to analyse the data is a comparison of the data taken in a high time resolution experiment with time dependent functions. High accuracy can be obtained for the determination of difference frequencies.

In this letter we present new experimental data of time-resolved coherent Raman spectroscopy taken with a novel femtosecond Raman spectrometer [12, 13]. The results of these time-domain experiments are subsequently numerically analysed. In this way we obtain frequency differences with high precision. The high time resolution of the femtosecond coherent Raman set-up allows one to measure very high difference frequencies. After a short theoretical description of the basic ideas of the technique we give experimental results. We show that terahertz quantum beats up to $10 \mathrm{THz}$ can be measured giving precise values for the frequency differences of vibrational transitions separated by up to $350 \mathrm{~cm}^{-1}$.

\section{Theory.}

Time-resolved coherent Raman scattering is commonly treated under the following assumptions [1]. The light fields are described by Maxwell's equation 
and the vibrational transitions are represented by two-level systems. Changes in the population of the two molecular levels are neglected (weak Raman interaction). The expectation value of the vibrational amplitude, the coherent amplitude $\langle q\rangle$, is the relevant quantity for the description of time-resolved coherent Raman spectroscopy. First, we discuss the coherent experiment for a single homogeneously broadened transition with dephasing time $T_{2}$. We use the ansatz of plane waves for the coherent amplitude $\langle q\rangle=(i / 2) Q \exp \left(-i \omega_{q} t+i k_{q} x\right)+$ c.c. and assume an isotropic Raman tensor for the investigated vibration. The coherent amplitude of a Raman active mode at frequency $\omega_{q}$ is excited via transient stimulated Raman scattering by a pair of light pulses, the laser pulse $E_{\mathrm{L}}$ and the Stokes pulse $E_{\mathrm{S}}[1,14]$. The electric fields are considered to be plane waves, e.g.

$$
E_{\mathrm{L}}=1 / 2 E_{\mathrm{L}} \exp \left(-i \omega_{\mathrm{L}} t+i k_{\mathrm{L}} x\right)+\text { c.c. }
$$

with the wave vector $k_{\mathrm{L}}$ and frequency $\omega_{\mathrm{L}}$ for the laser field. The driving force $F(x, t)$ for the coherent amplitude $Q$ is proportional to the product of the laser and the Stokes field, $F(x, t) \propto E_{\mathrm{L}} E_{\mathrm{S}}^{*}$; as a result the frequency of the excitation force is $\omega_{L}-\omega_{S}$. The linear response theory applied to the excitation process leads to the following equation for the coherent amplitude $Q$ :

$$
\begin{aligned}
& Q(t)=\kappa \int_{-\infty}^{t} \mathrm{~d} t^{\prime} E_{\mathrm{L}}\left(t^{\prime}\right) E_{\mathrm{S}}^{*}\left(t^{\prime}\right) \times \\
& \times \exp \left[\left(t^{\prime}-t\right) / T_{2}-i \Delta \omega t^{\prime}\right] .
\end{aligned}
$$

The constant $\kappa$ contains material parameters such as Raman cross-section and vibrational frequency $\omega_{q}$ (see Ref. [1]). $T_{2}$ stands for the dephasing time of the transition. Detuning between the excitation frequency and the vibrational mode is given by $\Delta \omega=\omega_{\mathrm{L}}-\omega_{\mathrm{S}}-\omega_{q}$. Under the action of a short exciting force the coherent amplitude $\langle q\rangle$ evolves as follows : $\langle q\rangle$ rises to a maximum with the exciting force and, subsequently, decays exponentially with the dephasing time $T_{2}$. During the free exponential decay the molecules oscillate at the resonance frequency $\omega_{q}$. In the experiment described here the large bandwidth of the short driving force allows several vibrations to be excited simultaneously. Since the excitation via stimulated Raman interaction is weak, the various modes evolve in time independent of each other. The different vibrations may be treated with the same ansatz introducing the individual resonance frequencies $\omega_{q j}$, phase factors $\phi_{j}$, amplitudes $Q_{j}$, and dephasing times $T_{2 j}$.

In the time-resolved coherent Raman experiments, the amplitude $\langle q\rangle$ generated at time zero is monitored by coherent Raman scattering of a delayed probing pulse $E_{\mathrm{L} 2}$. We investigate light on the anti-Stokes side of the Raman spectrum generated by the nonlinear polarization $P_{\mathrm{AS}}^{\mathrm{NL}}$ :

$$
P_{\mathrm{AS}}^{\mathrm{NL}}=\lambda\langle q\rangle E_{\mathrm{L} 2}+\chi_{\mathrm{NR}}^{(3)} E_{\mathrm{L}} E_{\mathrm{S}}^{*} E_{\mathrm{L} 2} .
$$

The coherent signal consists of two contributions. The first part on the r.h.s. of equation (2) describes the coherent Raman signal of interest scattered from the excited vibrational mode. The constant $\lambda$ contains the Raman cross-section of the mode. This part of the coherent signal is emitted at the resonant antiStokes frequency $\omega_{\mathrm{AS}}=\omega_{\mathrm{L}_{2}}+\omega_{q}$ and provides information on the dynamic properties of the investigated mode. The second part on the r.h.s. of equation (2) is related to the nonresonant nonlinear susceptibility $\chi_{\mathrm{NL}}^{(3)}$ resulting from electronic contributions [15]. The nonresonant part of the signal gives an instantaneous response, i.e. it follows the product $E_{\mathrm{L}} E_{\mathrm{S}}^{*} E_{\mathrm{L} 2}$ and decays very rapidly with the time resolution of the experiment. The resonant part, on the other hand, shows the slow response of the resonantly excited vibrations. The nonresonant part of the coherent signal does not provide any information on the investigated modes. It has to be separated from the resonant signal. This fact is not possible in the steady-state coherent anti-Stokes Raman spectroscopy (CARS). In the time-resolved coherent anti-Stokes experiments described here the separation is possible due to the different time dependence of the two contributions: the rapidly decaying nonresonant part shows up around time zero, while at later delay times valuable information on the slower resonant part of the signal is collected.

In the present coherent experiment we measure the scattered Raman light as a function of the time delay $t_{\mathrm{D}}$ between excitation and probing pulses. In order to have optimum temporal resolution, pulses of very short duration for the exciting and probing laser fields are required.

Under these conditions the nonresonant signal is concentrated only around time zero and may be neglected at later times. Experimentally, one detects the time-integrated coherent signal $S\left(t_{\mathrm{D}}\right)$ :

$$
\begin{aligned}
S\left(t_{\mathrm{D}}\right) & \propto \int_{-\infty}^{+\infty} \mathrm{d} t\left|E_{\mathrm{AS}}\left(t, t_{\mathrm{D}}\right)\right|^{2} \\
& \propto \int_{-\infty}^{+\infty} \mathrm{d} t\left|P_{\mathrm{AS}}^{\mathrm{NL}}\left(t, t_{\mathrm{D}}\right)\right|^{2} \\
& \simeq \int_{-\infty}^{+\infty} \mathrm{d} t\left|\lambda\langle q\rangle E_{\mathrm{L} 2}\right|^{2} .
\end{aligned}
$$

According to equation (3) one measures the time dependence of the coherent amplitude or - more exactly - one determines the absolute square of the coherent amplitude, which decays exponentially with $T_{2} / 2$.

When several modes are excited simultaneously, the signal decay is more complex. It can be shown 
that for very short driving and probing pulses the coherent signal is equivalent to the absolute square of the Fourier transform of the spontaneous Raman spectrum [6, 7]. Here we analyse the coherent Raman signal at late delay times, where the different vibrational modes oscillate at their resonance frequencies $\omega_{q j}$ and the amplitudes decay exponentially with the individual dephasing times $T_{2 j}$. The coherent anti-Stokes field is the sum of the light field generated by the individual molecular modes $Q_{j}$. For short light pulses of duration $t_{\mathrm{p}} \ll T_{2}$, $t_{\mathrm{p}} \ll 1 / \Delta \omega_{i j}$ one obtains :

$$
\begin{aligned}
E_{\mathrm{AS}}\left(t, t_{\mathrm{D}}\right) & =\sum_{j} E_{\mathrm{AS} j}\left(t, t_{\mathrm{D}}\right) \\
& \propto \sum_{j} Q_{j}\left(t_{\mathrm{D}}\right) \exp \left(-i \omega_{j} t_{\mathrm{D}}\right) .
\end{aligned}
$$

According to equations (3) and (4) simultaneously excited modes lead to a beating of the coherent signal at the frequency differences $\Delta \omega_{i j}$ between the various transition frequencies $\Delta \omega_{i j}=\omega_{i}-\omega_{j}$. These « quantum beats » of the coherent signal may give accurate information on the vibrational spectrum.

The effect of different parameters on the coherent signal is readily seen for the simple example of two vibrational modes. We assume that the two modes with a frequency difference $\Delta \omega$ and dephasing times $T_{21}$ and $T_{22}$ are excited to the coherent amplitude $Q_{j 0}$. For equal amplitudes $Q_{10}=Q_{20}$ one obtains for the coherent signal

$$
\begin{aligned}
& S\left(t_{\mathrm{D}}\right)= \\
& =\left|\sum_{j=1,2} Q_{j 0} \exp \left(-t_{\mathrm{D}} / T_{2 j}-i \omega_{j} t_{\mathrm{D}}\right)\right|^{2} \\
& =Q_{10}^{2}\left[\exp \left(-2 t_{\mathrm{D}} / T_{21}\right)+\exp \left(-2 t_{\mathrm{D}} / T_{22}\right)+\right. \\
& \left.\quad+2 \cos \left(\Delta \omega t_{\mathrm{D}}\right) \exp \left(-t_{\mathrm{D}}\left(T_{22}+T_{21}\right) / T_{21} T_{22}\right)\right]
\end{aligned}
$$

The signal consists of the two contributions of the individual transitions decaying with the respective dephasing times (first two expressions on the r.h.s. of Eq. (5)). The interference between the two molecular modes gives rise to the third term of equation (5), which causes strong modulation of the signal. When the two dephasing times strongly differ, the modulation depth of the signal decreases with increasing time. At late delay times the modulation dies out and a single unmodulated exponential decay appears. As an example, the signal curve for two vibrational modes at $\Delta \omega=10^{13} \mathrm{~s}^{-1}$ $\left(=53 \mathrm{~cm}^{-1}\right), \quad T_{21}=6 \mathrm{ps}$ and $T_{22}=2 \mathrm{ps}, \quad$ and $Q_{1}(0) / Q_{2}(0)=0.9$ is depicted in figure 1 . At early times, when the two coherent amplitudes $Q_{1}(t)$ and $Q_{2}(t)$ are not yet affected by the exponential decay, the signal is strongly modulated by the frequency difference $\Delta \omega$. At later times the more rapidly

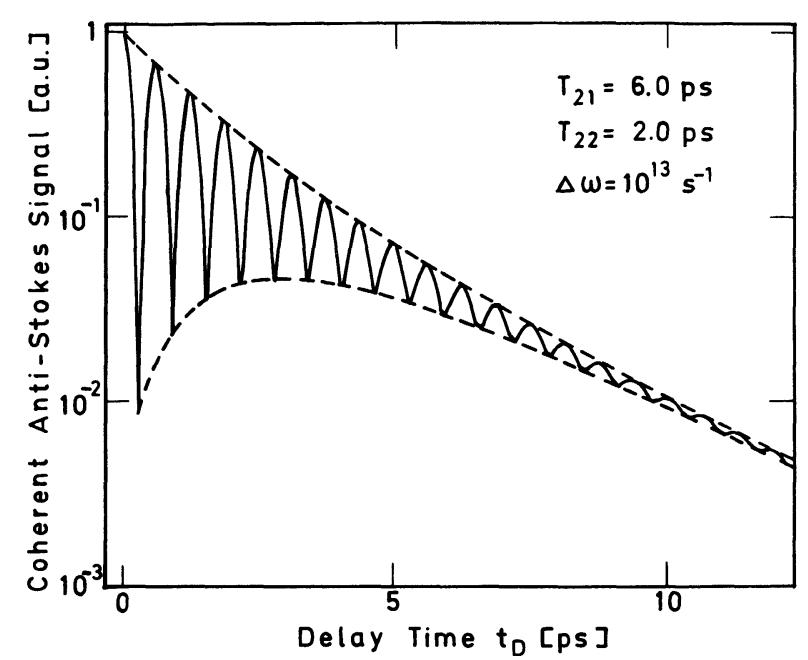

Fig. 1. - Coherent Raman signal calculated for two simultaneously excited molecular modes with the frequency difference $\Delta \omega=10^{13} \mathrm{~s}^{-1}\left(\triangleq 53 \mathrm{~cm}^{-1}\right)$. The initial amplitudes have a ratio of $Q_{10} / Q_{20}=0.9$ and dephasing times $T_{21}=6 \mathrm{ps}$ and $T_{22}=2 \mathrm{ps}$. The modulation of the signal decreases with the shorter one of the two dephasing times.

decaying coherent amplitude, and with it the modulation, vanishes. Data similar to figure 1 allow the determination of the two dephasing times and the frequency difference $\Delta \omega$.

For more complicated situations equations (3) and (4) permit the numerical analysis of the experimental data. With a least square-fit procedure one may deduce the molecular parameters such as dephasing times and frequency differences, by minimizing the difference between the experimental data and the theoretical curves. As shown below, the molecular quantities may be determined with high precision.

An alternative possibility to obtain the frequencydomain information from the time domain data is the straight forward application of a Fourier transformation [7]. As discussed in the literature it is advantageous to multiply the experimental data by a correction function, which compensates the exponential decay of the signal within the boundaries of the experimental time range. In this way the calculated line shape is not Lorentzian, and the accuracy of the line positions is improved.

\section{Experimental.}

The experimental system is depicted schematically in figure 2. Pulses from a mode-locked argon-ion laser synchronously pump two dye lasers. The specially designed femtosecond unidirectional ring (UDR) dye laser [16, 17] contains an amplifying jet (dye Rhodamine 6G) and an absorber jet (dye DODCI). The laser emits pulses with a duration of $80 \mathrm{fs}$ at $\lambda=625 \mathrm{~nm}$ with an average power of $30 \mathrm{~mW}$. The pulses from this laser provide us with the exciting 


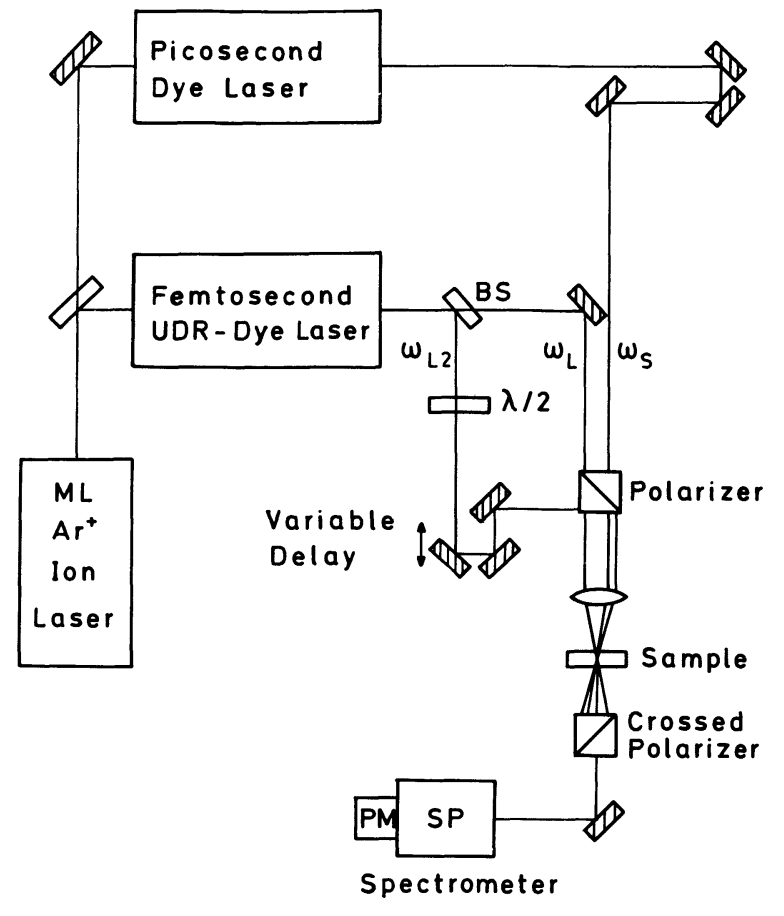

Fig. 2. - Experimental set-up used for Fourier transform coherent Raman spectroscopy. A mode-locked argon-ion laser synchronously pumps a femtosecond unidirectional ring dye laser (frequency $\omega_{\mathrm{L}}$ ) and a tunable picosecond dye laser (frequency $\omega_{\mathrm{S}}$ ). Excitation is done with a synchronized pair of pulses at $\omega_{\mathrm{L}}$ and $\omega_{\mathrm{S}}$, while the probing pulses at $\omega_{\mathrm{L} 2}$ are produced by a beam splitter BS from the femtosecond laser pulses $\omega_{\mathrm{L}}$. The coherent antiStokes light generated via the probing pulse is recorded as a function of the time delay adjusted by the variable delay line.

pulses $E_{\mathrm{L}}$ and, via the beam splitter $\mathrm{BS}$, with the probing pulses $E_{\mathrm{L} 2}$. The second laser is a standard synchronously mode-locked picosecond dye laser with amplifying dyes DCM or Pyridine 2 and a threeplate birefringent filter as a tuning element (power $30 \mathrm{~mW}$ ). When tuned through the red and the infrared part of the spectrum, pulses $E_{\mathrm{S}}$ at the Stokes frequency $\omega_{\mathrm{S}}$ of $6 \mathrm{ps}$ duration are generated. The femtosecond pulses from the UDR laser and the picosecond pulses of the second laser, both with parallel polarization, serve to excite molecular vibrations with a tuning range between $300 \mathrm{~cm}^{-1}$ and $3000 \mathrm{~cm}^{-1}$. It can be derived from equation (1) that the driving force exists only for the time duration of the shorter of the two pulses, i.e. for a time of $80 \mathrm{fs}$. A major advantage of the system is the wide tunability of the excitation frequency $\omega_{L}-\omega_{S}$ with the help of the birefringent filter of the picosecond laser while keeping the favorable femtosecond time resolution. The coherent vibrational excitation is monitored by probing pulses derived from the femtosecond laser. These pulses are polarized perpendicular to the excitation pulses. The three beams are focused into the sample using a geometry appro- priate for anti-Stokes phase matching. The antiStokes radiation $E_{\mathrm{AS}}$ generated by the probing process passes the second polarizer and is detected in conjunction with the broad-band spectrometer SP by the cooled photomultiplier PM. The coherent signal is recorded as a function of the delay time (set by the variable delay line) between the exciting and probing pulses.

\section{Fourier transform coherent Raman spectroscopy of transitions with terahertz frequency differences.}

High-resolution Fourier transform coherent Raman spectroscopy was first reported by Graener and Laubereau [7], who worked with light pulses of 20 ps duration. The authors studied vibrational-rotational transitions in $\mathrm{CH}_{4}$ separated by less than $1 \mathrm{~cm}^{-1}$ with high frequency resolution of $10^{-3} \mathrm{~cm}^{-1}$. The data were taken over a time interval of $12 \mathrm{~ns}$.In the experiment described here we extend Fourier transform coherent Raman spectroscopy for the first time to the study of large frequency differences up to $350 \mathrm{~cm}^{-1}$. To see the related beating phenomena one requires high time resolution. For example, a frequency difference of $350 \mathrm{~cm}^{-1}$ produces a beating pattern with peaks separated by $95 \mathrm{fs}$. In order to resolve this very rapid phenomenon, one has to work with femtosecond light pulses. Synchronized light pulses of approximately $80 \mathrm{fs}$ duration became available only recently $[16,17]$ permitting the experiments reported here.

The first investigation treats vibrations in neat pyridine and in pyridine/cyclohexane mixtures. We study Raman active vibrations of liquid pyridine at frequencies $991 \mathrm{~cm}^{-1}$ and $1030 \mathrm{~cm}^{-1}$ assigned to two $A_{1}$ ring modes [18]. Both vibrations have similar spectral width $\left(\sim 2.2 \mathrm{~cm}^{-1}\right)$ and similar Raman cross-sections ; they are separated by $39 \mathrm{~cm}^{-1}$. In the Raman excitation process a frequency difference of $\left(\omega_{\mathrm{L}}-\omega_{\mathrm{S}}\right) / 2 \pi c=1010 \mathrm{~cm}^{-1}$ between the laser and the Stokes frequency is applied. Due to the broad spectral width of the femtosecond exciting force of more than $200 \mathrm{~cm}^{-1}$ both pyridine modes are simultaneously excited in the experiment. Figure $3 \mathrm{a}$ shows the observed anti-Stokes signal plotted as a function of the time delay between exciting and probing pulses. During the excitation process at time zero the coherent signal rises to a pronounced peak. It subsequently decays quickly over more than one order of magnitude. Later on the signal recovers and shows strong oscillations. The modulation depths exceeds a factor of ten. Two features of the coherent signal are of special interest here : (i) the period of oscillation is approximately $0.85 \mathrm{ps}$. Consequently, the frequency difference between the two modes is $1.18 \mathrm{THz}$. (ii) The peaks of the oscillation decay exponentially with the decay time $T_{2} / 2=2.55 \pm 0.15 \mathrm{ps}$. The depth of the oscilla- 


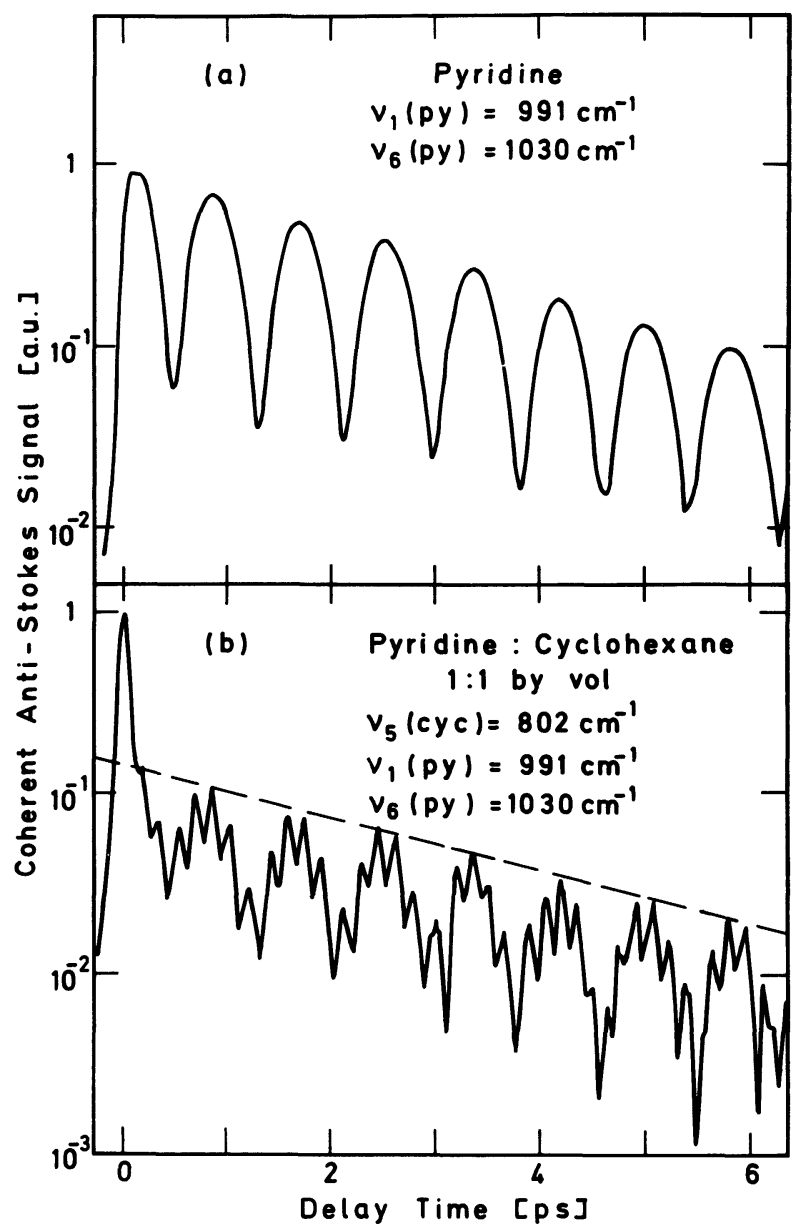

Fig. 3. - Time resolved coherent Raman scattering using femtosecond time resolution. (a) Beat pattern of pure pyridine after excitation of the two pyridine modes at $991 \mathrm{~cm}^{-1}$ and $1030 \mathrm{~cm}^{-1}$. (b) Beat pattern of a mixture of pyridine and cyclohexane. Three molecular modes are excited.

tion slightly decreases for long observation times indicating a difference between the dephasing times of the two modes of $10 \%$.

In figure $3 \mathrm{~b}$ we present an amazingly complicated, but perfectly reproducible anti-Stokes pattern. A mixture of cyclohexane and pyridine $(1: 1$ by volume) was investigated with femtosecond light pulses. In this case three vibrational modes with similar $T_{2}$ values beat together. They are : one cyclohexane mode at $802 \mathrm{~cm}^{-1}$ [19] and two pyridine modes at $991 \mathrm{~cm}^{-1}$ and $1030 \mathrm{~cm}^{-1}$. We find a rich beating structure originating from the interference of the three modes. The frequency differences of the excited modes are determined by the following procedure. The exponential decay is removed by multiplying the signal with an exponential rising function. An appropriate window function is introduced to remove the influence of the boundaries of the time range. After these arithmetical manipulations a Fourier transformation of the time-dependent data gives the results shown in figure 4 . Three

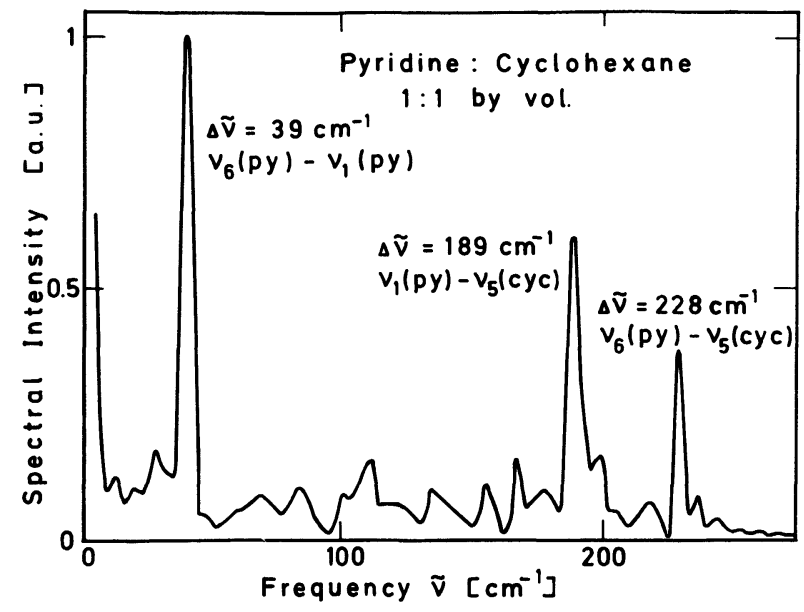

Fig. 4. - Difference spectrum of pyridine and cyclohexane obtained by numerical Fourier transformation of the time resolved data from figure $3 \mathrm{~b}$. Note the strong peaks at $39 \mathrm{~cm}^{-1}, 189 \mathrm{~cm}^{-1}$, and $228 \mathrm{~cm}^{-1}$, which correspond to the differences betweeen the three excited modes. The highest difference frequency is $\Delta \nu=6.84 \mathrm{TH} z$.

sharp and pronounced peaks are found at $39 \mathrm{~cm}^{-1}$, $189 \mathrm{~cm}^{-1}$ and $228 \mathrm{~cm}^{-1}$, which correspond to the frequency differences between the three modes excited in the mixture. The frequency width of the three different lines is approximately $5 \mathrm{~cm}^{-1} ;$ this width is due to the limited time range of observation (e.g. 6 ps in Fig. 3). The background noise in figure 4 reflects the experimental noise of figure 3 and the applied window function.

A very promising method to evaluate the experimental data is the modelling of the beat pattern according to equations (3) and (4). An example is given in figure $5 \mathrm{a}$, where we consider two modes to simulate an extended beating pattern of neat pyridine (redrawn in Fig. 5b for ready comparison). Two theoretical curves are calculated for the same set of experimental parameters. Only the difference frequency $\Delta \omega$ was changed by the small amount of $0.4 \mathrm{~cm}^{-1}$ (i.e. $1 \%$ ) from $39.2 \mathrm{~cm}^{-1}$ (broken curve) to $39.6 \mathrm{~cm}^{-1}$ (solid curve). Superposition of the experimental and calculated curves gives an impressive fit over the entire time range. It is possible to deduce a very precise frequency difference of $39.4 \pm 0.2 \mathrm{~cm}^{-1}$.

Interesting results were obtained in the coherent Raman study of liquid nitrobenzene, where we observe the highest beat frequencies. With an excitation frequency at $1200 \mathrm{~cm}^{-1}$ we detect a rich beating structure. In figure $6 \mathrm{~b}$ the coherent signal curve is plotted on a linear scale for a time interval from $0.2 \mathrm{ps}$ to $2.5 \mathrm{ps}$. An extremely rapid modulation is found with the shortest time between subsequent peaks of less than $100 \mathrm{fs}$. A Fourier transform to the frequency domain reveals four strong peaks at $16 \mathrm{~cm}^{-1}, 104 \mathrm{~cm}^{-1}, 237 \mathrm{~cm}^{-1}$, and $341 \mathrm{~cm}^{-1}$, and a 


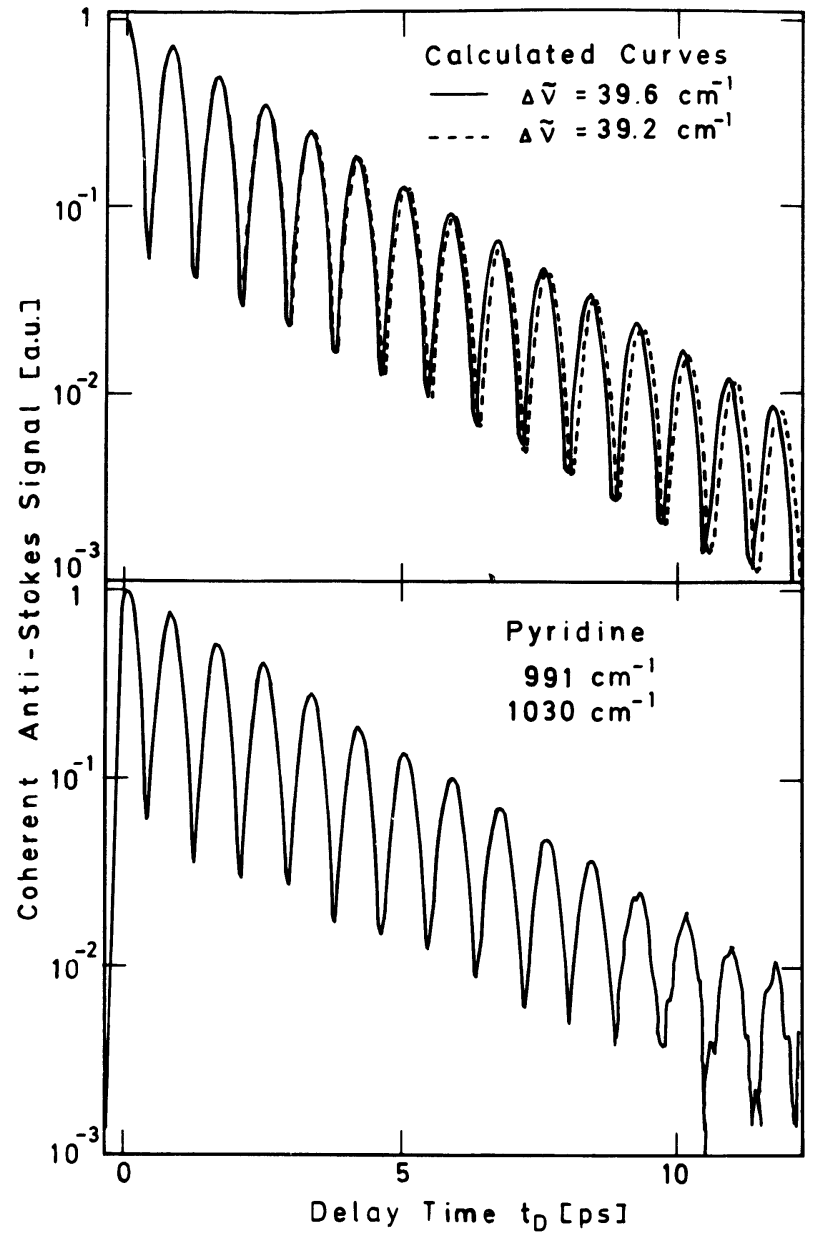

Fig. 5. - Time resolved coherent Raman data for liquid pyridine. (a) Two curves calculated according to equations (3) and (4) using the dephasing times $T_{21}$ $\left(991 \mathrm{~cm}^{-1}\right)=5.1 \mathrm{ps}$ and $T_{22}\left(1030 \mathrm{~cm}^{-1}\right)=4.7 \mathrm{ps}$ and the frequency differences of $39.6 \mathrm{~cm}^{-1}$ and $39.2 \mathrm{~cm}^{-1}$. Comparison of the calculated curves with the experimental data of figure $5 b$ gives a very accurate number for the frequency difference between the two modes of $\Delta \nu=$ $39.4 \pm 0.2 \mathrm{~cm}^{-1}$.

number of weaker peaks. The value of $341 \mathrm{~cm}^{-1}$ or $10.5 \mathrm{THz}$ corresponds to the beating of the two nitrobenzene modes at $1000 \mathrm{~cm}^{-1}$ and $1341 \mathrm{~cm}^{-1}$. Both modes were excited simultaneously in the transient stimulated Raman process. In figure 6a we show a beat curve calculated for the following set of data :

$$
\begin{aligned}
\nu / c=1000 \mathrm{~cm}^{-1}, & T_{2}=2.9 \mathrm{ps} \\
1016.4 \mathrm{~cm}^{-1}, & T_{2}=2.7 \mathrm{ps} \\
1104.2 \mathrm{~cm}^{-1}, & T_{2}=1.1 \mathrm{ps} \\
1340.9 \mathrm{~cm}^{-1}, & T_{2}=2.1 \mathrm{ps}
\end{aligned}
$$

Using a least square-fit procedure to adjust the theoretical parameters a very good agreement of the theoretical and experimental curves is achieved. The data analysing procedure indicates that the frequency

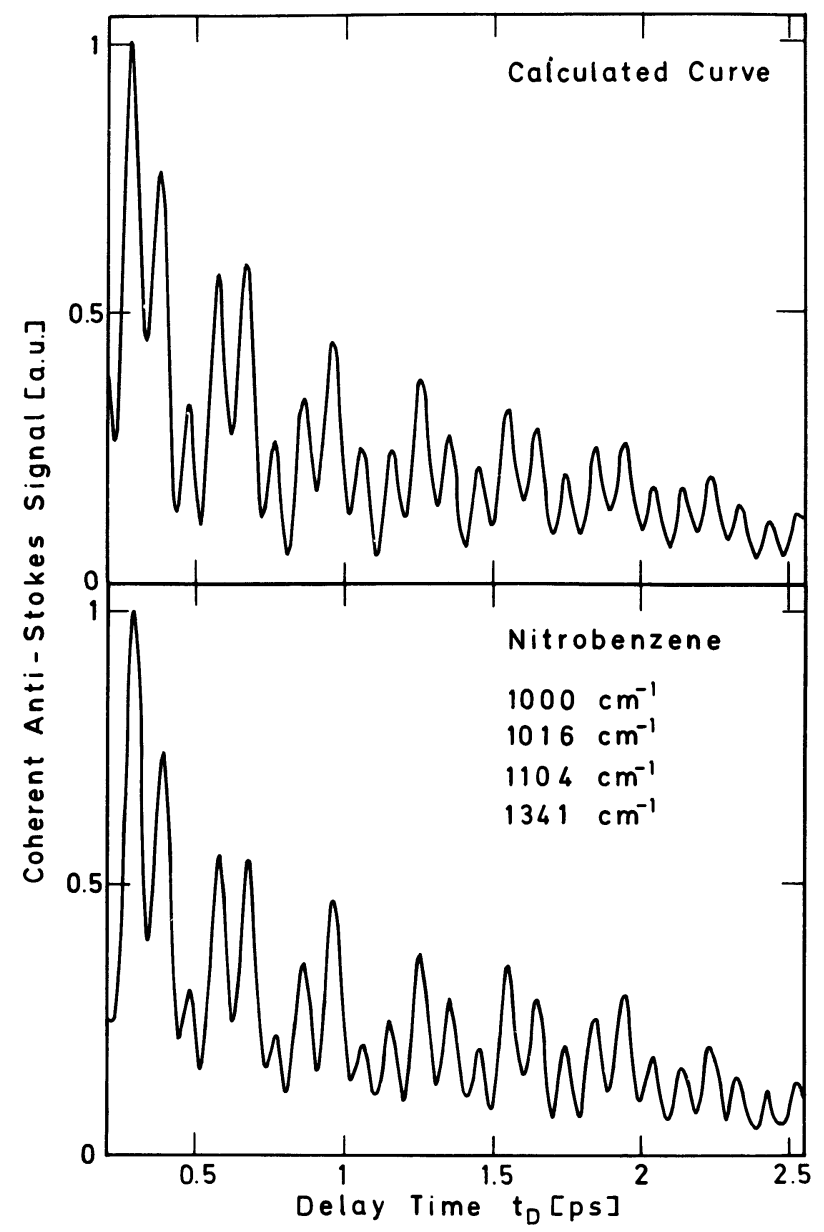

Fig. 6. - Time resolved coherent Raman data for liquid nitrobenzene. (a) The curve (calculated by a least squarefit procedure should be compared with the results shown below). The four vibrational components with difference frequencies $\Delta \nu / c$ up to $340.8 \mathrm{~cm}^{-1}$ are listed in figure $6 \mathrm{~b}$. (b) Experimental curve of liquid nitrobenzene showing high-frequency beat phenomena extending to frequencies of $10.5 \mathrm{THz}$.

differences $\Delta \Omega$ may be determined with a precision approaching $10^{-3}$.

\section{Summary.}

In this paper we have extended Fourier transform coherent Raman spectroscopy to the frequency range of $10 \mathrm{THz}$. With this technique we are now able to resolve terahertz phenomena with high accuracy.

Two points are relevant : (i) the frequency resolution is determined by the total time interval of the experiment. In order to improve spectral resolution the coherent signal has to be measured over delay times as long as possible. Taking into account the exponential decay of the signal with $T_{2} / 2$ one immediately finds that the spectral resolution is directly related to the signal-to-noise ratio of the experiment. (ii) The duration of the exciting and 
probing pulses does not influence the frequency resolution of the experiment, but determines the highest detectable beat frequencies to approximately
$\Delta \nu_{\max } \simeq 1 / t_{\mathrm{p}}$. Under the present experimental conditions beat frequencies exceeding $10 \mathrm{THz}$ are readily detected.

\section{References}

[1] Laubereau, A., Kaiser, W., Rev. Mod. Phys. 50 (1978) 607 ;

Penzkofer, A., Laubereau, A., Kaiser, W., Progr. Quantum. Electron. 6 (1979) 55.

[2] Von der Linde, D., Laubereau, A., Kaiser, W., Phys. Rev. Lett. 26 (1971) 954.

[3] Velsko, S., Trout, J., Hochstrasser, R. M., J. Chem. Phys. 79 (1983) 2114.

[4] Gale, G. M., Guyot-Sionnest, P., Zheng, W. Q. Opt. Commun. 58 (1986) 395.

[5] D'yakov, Yu. S., KRIKunov, S. A., Magnitskil, S. A., Nikitin, S. Yu., Tunkin, V. G., Sov. Phys. JETP 57 (1983) 1172.

[6] ZinTH, W., KAISER, W., In Organic Molecular Aggregates, Eds R. Reinecker, H. Haken, H. C. Wolf, Solid State Science, Vol. 49 (Springer, New York) 1983, p. 124.

[7] Graener, H., Laubereau, A., Opt. Commun. 54 (1985) 141.

[8] Zinth, W., Opt. Commun. 34 (1980) 479.

[9] Zinth, W., Nuss, M. C., Kaiser, W., Chem. Phys. Lett. 88 (1982) 257.
[10] Collins, M. A., Madden, P. A., Buckingham, A. D., Chem. Phys. 94 (1985) 291.

[11] Zinth, W., Nuss, M. C., Kaiser, W., Phys. Rev. A 30 (1984) 1139.

[12] LeonhardT, R., Holzapfel, W., Zinth, W., KaISer, W., Chem. Phys. Lett. 133 (1987) 373.

[13] Zinth, W., LeOnhardt, R., Holzapfel, W., KAISER, W., submitted to J. Quantum Electron.

[14] Carman, R. L., Shimizu, I., Wang, C. S., BloemBergen, N., Phys. Rev. A 2 (1970) 60.

[15] Zinth, W., Laubereau, A., Kaiser, W., Opt. Commun. 26 (1978) 457.

[16] Nuss, M. C., LeOnhardT, R., Zinth, W., Opt. Lett. 10 (1985) 16.

[17] Dobler, J., Schulz, H. H., Zinth, W., Opt. Commun. 57 (1986) 407.

[18] Long, D. A., Murfin, F. S., Thomas, E. L., Trans. Farad. Soc. 59 (1963) 12.

[19] Wiberg, K. B., Shrake, A., Spectrochim. Acta 27A (1971) 1139. 